\section{KENTRON}

REVUE PLURIDISCIPLINAIR

DU MONDE ANTIQUE

\section{Kentron}

Revue pluridisciplinaire du monde antique

$20 \mid 2004$

La démesure

\title{
Franco Maiullari, Omero anti-Omero. Le incredibile storie di un trickster giullare alla corte micenea
}

\section{François Jouan}

\section{(2) OpenEdition}

\section{Journals}

Édition électronique

URL : http://journals.openedition.org/kentron/1834

DOI : 10.4000/kentron.1834

ISSN : 2264-1459

Éditeur

Presses universitaires de Caen

\section{Édition imprimée}

Date de publication : 31 décembre 2004

Pagination : 190-193

ISBN : 2-84133-251-9

ISSN : 0765-0590

\section{Référence électronique}

François Jouan, «Franco Maiullari, Omero anti-Omero. Le incredibile storie di un trickster giullare alla corte micenea », Kentron [En ligne], 20 | 2004, mis en ligne le 09 avril 2018, consulté le 19 novembre 2020. URL : http://journals.openedition.org/kentron/1834 ; DOI : https://doi.org/10.4000/kentron.1834

\section{(c) (i) (9)}

Kentron is licensed under a Creative Commons Attribution-NonCommercial-NoDerivatives 3.0 International License. 
donne en fait quatre contributions indépendantes consacrées à des mythes d'initiation, dans lesquels se retrouve évidemment tel ou tel des dix-sept thèmes. La même remarque vaut pour la troisième partie, "Ulysse : une vie d'épreuves initiatiques ", p. 161-198. Mais, s'agissant de la collation d'articles déjà publiés, c'est la loi du genre. Les quatre articles de la deuxième partie sont: «Le labyrinthe et la grue » (p. 59-86; mythe de Thésée) ; «Lupus duplex» (p. 87-127; figures et mythes du loup, de Lycomède à Lycurgue) ; «Odyssée, XI, 101-139: l'examen de passage de Télémaque» (p. 129140); «L'Oreste d'Eschyle: un justicier ou un initié?» (p. 141-160).

Signalons que l'ouvrage se termine par une bibliographie très complète sur l'initiation (p. 201-245), dont la recension s'étend jusqu'en 2002.

Bernard Deforge

\section{Franco Maiullari, Omero anti-Omero. Le incredibile storie di un trickster giullare alla corte micenea, Rome, Edizioni dell'Ateneo (Filologia e Critica;} 91), 2004, $271 \mathrm{p}$.

Nos lecteurs, auxquels on a rendu compte à plusieurs reprises des travaux de Franco Maiullari, helléniste et psychothérapeute, Suisse de culture italienne, savent qu'il professe sur les Tragiques grecs des thèses qui ne s'embarrassent pas de la stricte orthodoxie. Ce n'est pas avec moins de liberté d'esprit qu'il aborde dans ce livre les épopées homériques, et le moins qu'on puisse dire, c'est que l'image traditionnelle du Poète tel que l'ont vénéré les Anciens n'en sort pas intacte. Mais peut-être que, descendu de son Olympe pour devenir un batteur d'estrade, un « jongleur » à la cour des seigneurs de Mycènes, l'Homère de F. Maiullari, pour les lecteurs qu'il aura convaincus, aura gagné en familiarité humaine ce qu’il aura perdu en majesté !

Il n'est pas aisé de rendre compte d'un livre fourmillant d'idées, mais où cellesci comportent des enchaînements parfois imprévisibles, et où les réminiscences perturbent l'ordre formel en parties et en chapitres. Essayons cependant de définir les grands traits de la physionomie d'Homère, tels qu'ils ressortent du livre : l'auteur de l'Iliade et de l'Odyssée (le même poète, et sur ce point F.M. apporte des arguments non négligeables) n'est pas seulement le chantre qui exalte les hauts faits des temps anciens, célébrant ses héros et ses dieux, mais aussi un esprit critique et malicieux, qui mêle au sérieux de ses récits des touches de scepticisme et d'ironie. À cet égard, Homère apparaitrait comme le créateur d'un genre littéraire auquel les Anciens ont donné le nom de spoudaiogéloion, le sérieux-plaisant. Seule la lecture anamorphique des poèmes, à laquelle F.M. a initié ses lecteurs, permet de déceler les équivoques volontaires que l'aède sème dans son récit. Si l'on admet ce caractère des épopées homériques, on comprend mieux que l'auteur de l'Iliade et de l'Odyssée ait pu être 
considéré aussi dans l'Antiquité comme le créateur du Margitès et de la Batrachomyomachie.

Trois adjectifs sont utilisés par F.M. pour qualifier les épopées d'Homère : elles sont oniriques, cycliques et parodiques. C'est ce qui est exposé dans la première partie du livre, et développé plus largement dans la seconde. Je passerai rapidement sur l'appellation de cyclique, parce que c'est elle qui me parait la moins bien établie. Elle se fonde surtout sur les débuts de l'Iliade. L'auteur montre très justement que le poète n'organise pas son récit suivant la chronologie, mais de façon à mettre en relief des motifs qui alternent, formant comme des cercles concentriques : l'humiliation qui produit la colère (mènin), et la colère qui suscite la vengeance. Ainsi, humiliation de Chrysès, colère d'Apollon, humiliation d'Agamemnon, qui humilie à son tour Achille, colère d'Achille qui fait appel à Thétis, recours de celle-ci à Zeus, qui va humilier Agamemnon en lui envoyant un songe trompeur, etc. Le discrédit d'Agamemnon atteint son comble avec l'échec de l'Ambassade. Mais l'analyse ne va guère plus loin, en sorte qu'on n'est guère prêt à accepter la définition proposée du poème comme "un récit onirique à cercles concentriques», non plus que le double schéma (p. 50-51) qui met en parallèle «les développements cycliques de l'Iliade» et le bouclier circulaire d'Achille du chant 18.

C'est encore au début du chant 2, avec le rêve trompeur d'Agamemnon, auquel F.M. donne un grand rôle, qu'apparaît sans conteste l'élément onirique. Mais il considère qu'on peut aussi qualifier de songes des scènes de vision éveillée qui présentent des caractères communs, et correspondent en général à la rencontre d'un mortel et d'une divinité: Chrysès et Apollon, Achille et Thétis. Les autres exemples de rêves sont peu nombreux: le songe prémonitoire de Rhésos au chant 11, celui que Zeus envoie à Priam au chant 24 ; mais d'autres scènes présentent des caractères oniriques, comme l'enlèvement de Pâris ou d'Énée sur le champ de bataille, l'apparition de la psychè de Patrocle au chant 23, l'intervention d'Hermès auprès de Priam au chant 24. Plusieurs fois il est fait référence aux interprètes des songes (oneiropoloi). Dans l'Odyssée, les songes proprement dits sont plus fréquents : ceux de Pénélope au chant 4 et au chant 19, celui de Nausicaa au chant 6 , et tout le début du chant 23 où alternent, aux deux étages du palais, les songes et les rêveries nocturnes des époux encore séparés, conclus par le clèdôn de l'esclave auprès de la meule. Comme dans l'Iliade, certains épisodes se déroulent dans un climat onirique, comme l'apparition d'Ino au chant 5, ou l'ensemble de la Nékyia. De l'analyse détaillée du chant 11, F.M. conclut qu'il porte tout entier le caractère d'une expérience onirique, dans laquelle défilent des visions étranges et incohérentes. Dans ce domaine aussi, le retour des mêmes motifs renforce la présomption d'un auteur unique des deux poèmes.

C'est au troisième thème, celui de la «parodie» (l'auteur reconnaît qu'il emploie ce terme faute de mieux), qu'est réservée la plus grande place. Dans le cours d'un récit empreint de gravité, il arrive que le poète se parodie en quelque sorte lui-même 
(d'où le titre du livre), laissant entendre à ses auditoires des cours seigneuriales qu'il ne prend au sérieux ni les exploits des héros ni même les interventions des dieux. Ainsi, nous l'avons vu, les débuts de l'Iliade jettent le discrédit sur le roi Agamemnon. Avec le songe mensonger, celui-ci est dupé par Zeus, qui agit comme un vrai trickster, un filou, en lui garantissant faussement la victoire si longtemps espérée. Entre Zeus, Thétis et Achille se noue un complot muet, sous le signe de l'omertà, puisqu'il est sanctionné par le seul signe de tête de Zeus (neusé, 1, 528). La trahison est partout, dans le trio humain Pâris-Hélène-Ménélas comme dans le trio divin Zeus-Héra-Thétis. Achille, d'abord présenté sous un jour favorable, manifeste lors de l'Ambassade un entêtement déraisonnable, puéril. Plus tard, il déchaîne sa férocité contre ses ennemis, avant de revenir à de meilleurs sentiments envers Priam. Athéna déploie à l'occasion une méchanceté quasi sadique. Aphrodite et Arès se ridiculisent lorsqu'ils se mêlent aux combattants. Après le duel peu glorieux de Pâris et de Ménélas, les dieux se montrent moins raisonnables que les hommes en rompant traîtreusement la trêve. Il y a beaucoup de futilité et de confusion dans les empoignades divines des chants 20 ou 21, tandis que les hommes qui se jettent en masse les uns contre les autres, sont comparés par Homère tantôt à des vols d'oiseaux, tantôt à des nuées de mouches. Et Zeus se contente d'en rire $(21,587)$.

Mais celui qui exprime le mieux la dérision de l'idéal héroïque, c'est Thersite, qui prend dans le livre une place (p. 75-85 et 167-188) bien supérieure à celle que lui accorde l'Iliade. Apparenté au mendiant Iros de l'Odyssée, au dieu du sarcasme, Mômos, il incarne le type populaire du bouffon, du gélôtopoios ou du trickster. Physiquement disgracié, il compense cette faiblesse par son insolence, une verve mordante qui s'en prend aux puissants et déclenche le rire de la foule. Il aura une belle postérité, depuis Margitès, les bouffons des banquets de Xénophon, de Lucien, de Plutarque, ceux des souverains hellénistiques et des empereurs romains, sans parler des cours royales d'Occident jusqu'à la Renaissance. Pour faire rire, il doit prendre le contre-pied de la réalité, dépeindre le «monde à l'envers » qui se cache derrière la société des héros. F.M. estime qu'Homère a voulu comprimer dans les cinquante jours de l'Iliade dix ans de combats autour du rocher d'Ilion pour en marquer la vanité, et il énumère toute une série d'épisodes dont on peut faire une lecture soit sérieuse, soit plaisante. Déjà dans l'Iliade, mais surtout dans l'Odyssée, Ulysse a bien des traits du trickster: un penchant irrésistible à la tromperie et au mensonge, un individualisme exacerbé, un nomadisme qui lui interdit de se fixer, même dans sa patrie. Aussi finira-t-il sa vie dans un «monde à l'envers », où on prend la rame du marin pour une pelle à vanner ! À la fois dans l'onirisme et dans la parodie de l'épopée, le clinicien de la psychè retrouve le dérangement d'un monde de l'illogisme et de l'incohérence : celui de Circé et des Lestrygons sera aussi bien celui des Oiseaux d'Aristophane que de la bureaucratie de Kafka. 
Certes, il y a là beaucoup d'idées ingénieuses et stimulantes, mais on peut se demander dans quelle mesure elles nous permettent d'approcher la physionomie d'Homère et d'éclairer sa "poétique». C'est l'objet de la synthèse offerte par les quarante dernières pages du volume. Il n'est pas douteux, pour l'auteur, que le Poète était un aède aveugle: dans l'Odyssée, le devin aveugle Démodocos l'emporte en tous points sur le devin voyant Phémios. Toute une tradition mythique accorde du reste la vision de l'esprit à celui qui est privé de la vue. Il n'a pu recevoir que dans une école la formation rhétorico-sophistique qui lui a permis d'acquérir la capacité d'imaginer de beaux récits, susceptibles de multiples variations, l'aptitude à saisir les désirs de son auditoire pour atteindre une subtile combinaison du sérieux et du plaisant, enfin la maîtrise technique du chant et de la musique. Tout cela ne s'est pas fait en un jour, mais au cours du temps l'aède a fini par gagner le respect des grands, par imposer sa place, sinon à la cour des rois mycéniens, au moins dans les palais de ceux qui se disaient leurs descendants. Membre d'une confrérie qui peut rivaliser avec d'autres (voir le concours poétique de Chalcis des Travaux et des Jours), c'est un artisan comme d'autres, spécialiste du chant et du récit. Aussi, on lui demande d'être, non pas véridique, mais vraisemblable et plaisant, ce qui laisse du champ à l'onirisme. F.M. rappelle le jugement avancé dans le Traité du Sublime: «Les récits homériques sont à prendre comme des songes envoyés par Zeus.» Dion Chrysostome, dans le Trö̈cos, parlait purement et simplement de mensonges. La moquerie à l'égard des héros, et même des dieux, est une des composantes de l'art de l'aède. Avec le temps, la poésie homérique sera sacralisée, mais non sans scandaliser certains (de Platon... à Zoïle, «le fouet d'Homère », et à Dion).

On saura gré à F.M. d'avoir apporté avec tant de verve sa contribution personnelle à un procès toujours ouvert. Il l'a fait avec une parfaite connaissance des épopées et de la « littérature» critique (ce dont témoigne une bibliographie ramassée, mais répondant bien au sujet). On pourra en contester tel point de détail, voire minimiser la part de la «parodie» (ou de l'onirisme) dans l'ensemble de l'œuvre homérique, mais on ne pourra minimiser la force et la cohérence de son argumentation.

Il faut enfin ajouter que de temps à autre (exactement dix-huit fois) F.M. coupe son exposé par ce qu'il appelle des incises de dimension variable (de quelques lignes à deux grandes pages). Elles concernent tel point particulier des poèmes, ou elles ouvrent sur une échappée vers d'autres champs littéraires (de Gilgameš aux tragiques grecs, à Shakespeare, au théâtre chinois ou aux auteurs français du XviI siècle), ou vers tel problème de caractère général, et ce ne sont sûrement pas les pages les moins stimulantes d'un ouvrage rafraîchissant par son non-conformisme.

François JouAN 